\title{
http://revistainvestigacionacademicasinfrontera.com
}

Recibido el 18 de marzo de 2018. Dictaminado mediante arbitraje favorablemente 2 de julio de 2018

\section{Interpretación de los discursos en torno a las disposiciones hídricas para comprender la demanda de abastecimiento en una localidad del centro de México}

Interpretation of the discourses around the water provisions to understand the demand for supply in a locality of central Mexico

José Marcos Bustos-Aguayo ${ }^{1}$, Michiko Amemiya-Ramírez ${ }^{2}$, Oscar Valdés-Ambrosio ${ }^{3}$, Cruz García-Lirios ${ }^{4}$

\section{Resumen}

Las disposiciones entendidas como aprendizajes para la optimización de recursos ante una situación que es percibida como abundante o escasa fueron interpretadas en un estudio cualitativo con tres informantes claves dedicados a la comercialización del agua. La develación del significado del aprendizaje de la optimización fue el aporte del presente trabajo, aún y cuando la literatura advierte que las diferencias prevalecen entre autoridades y usuarios. Pues bien, en el caso de los entrevistados la simbolización del agua como un bien comercial develó un significado de aprendizaje del ahorro del recurso. En tal sentido, la literatura advierte la importancia de encontrar diferencias y similitudes con respecto a las problemáticas hídricas de acuerdo con la zona urbana.

Palabras clave-Escasez, desabastecimiento, optimización, significado, discursos

\footnotetext{
1 UNAM, Facultad de Psicología: marcos.bustos@unam.mx

2 UNAM, Facultad de Arquitectura: amemiya@unam.mx

3 UNAM, Facultad de Economía: oscarva@unam.mx

4 UAEMEX, Facultad de Ciencias Políticas: cgarcial213@profesor.uaemex.mx
} 


\title{
$\underline{\text { http://revistainvestigacionacademicasinfrontera.com }}$
}

\begin{abstract}
The provisions understood as learning for the optimization of resources in a situation that is perceived as abundant or scarce were interpreted in a qualitative study with three key informants dedicated to the commercialization of water. The unveiling of the meaning of the learning of the optimization was the contribution of the present work, even though the literature warns that the differences prevail between authorities and users. Well, in the case of the interviewees, the symbolization of water as a commercial asset revealed a meaning of learning about resource saving. In this regard, the literature warns of the importance of finding differences and similarities with regard to water issues according to the urban area.
\end{abstract}

Keywords -Shortage, shortage, optimization, meaning, discourses

\section{Introducción}

El objetivo del presente trabajo fue develar el significado de las disposiciones hídricas en relación con las demandas de abastecimiento locales, considerando la escasez, insalubridad y carestía del servicio público en una demarcación de bajo desarrollo humano de la Ciudad de México.

Una disposición hídrica, para los fines del presente trabajo, es una serie de respuestas heredadas y aprendidas respecto a contingencias del entorno personal como es el caso de la difusión informativa de escasez y la experiencia de desabastecimiento de agua orientadas por la transferencia de conocimientos y habilidades de ahorro de agua (García, 2012). O bien, si los medios de comunicación promocionan una abundancia de agua reflejada en una alta calidad, entonces más bien se generan disposiciones a favor 


\section{http://revistainvestigacionacademicasinfrontera.com}

del consumismo debido a un contexto de oferta, recreación y confort (Carreón et al., 2011).

Precisamente, en el segundo caso donde las autoridades sustentan sus políticas de oferta acorde a la difusión de abundancia de agua se genera una relación de dependencia con los usuarios a través de subsidios y condonaciones en detrimento del aumento de tarifas en zonas no adherentes, simpatizantes o militantes al partido del gobierno o la oposición (Carreón et al., 2014).

En ese clima de representaciones de abundancia o escasez de agua los actores políticos y sociales se debaten entre el desabastecimiento o la regularidad del suministro (García et al., 2013), entre la insalubridad propiciada por las enfermedades hidro-transmitidas y la sanidad preventiva, entre la carestía como política local y la condonación del servicio por la militancia política (García, 2005).

Pues bien, los estudios de la gobernanza de la sustentabilidad hídrica local han advertido que son las disposiciones las que preceden al clima simbólico en el que las representaciones sociales son el inicio de un fenómeno (García, 2013). Además, esta relación entre representaciones y disposiciones predice acciones de derroche o ahorro de agua (García, 2006).

\section{Estudios de las disposiciones hídricas}

La Tabla 1 muestra los estudios de las disposiciones hídricas que se han centrado en la predicción del comportamiento proambiental (García et al., 2016), indicador de estilos de vida austeros y frugales con orientación a la sustentabilidad (García et al., 2017), la afinidad por la naturaleza y altruismo biosférico (García et al., 2015). 


\section{http://revistainvestigacionacademicasinfrontera.com}

Tabla 1. Estudios de las disposiciones hídricas

\begin{tabular}{|c|c|c|}
\hline Año & Autor & Determinantes \\
\hline 1980 & Berk et al., & Autointerés inmediato \\
\hline 1983 & Hamilton & Motivos idealistas \\
\hline 1986 & Corral et al., & Ahorro \\
\hline 1987 & Corral et al., & Refrigerador \\
\hline 1987 & Corral, Garibaldi y Encinas & Consumo doméstico \\
\hline 1992 & Corral y Obregón & Competencias y motivos \\
\hline 1992 & Corral y Obregón & CPA \\
\hline 1994 & Aitken et al., & Disonancia cognitiva \\
\hline 1997 & Corral & Actitud \\
\hline 1997 & Corral et al., & NPA \\
\hline 1998 & Corral & Interacciones \\
\hline 1999 & Bechtel, Corral y Pinheiro & Creencias \\
\hline 1999 & Corral y Pinheiro & Situacionales \\
\hline 1999 & De Oliver & Restricción hídrica \\
\hline 2000 & Bustos & Calidad \\
\hline 2000 & Corral & CPA \\
\hline 2000 & Corral y Zaragoza & Sociodemográficas \\
\hline 2000 & Corraliza y Martín & Actitudes \\
\hline 2000 & Obregón y Zaragoza & Tradiciones \\
\hline 2001 & Corral & Desabastecimiento \\
\hline 2001 & Corral & Modelamiento \\
\hline 2001 & Corral & Situacionales demográficas \\
\hline 2001 & Corral, Frías y González & Anti-ambiental \\
\hline 2001 & Van Vugt & Identidad \\
\hline 2002 & Bustos et al., & Motivos \\
\hline 2002 & Bustos, Flores y Andrade & Motivos y Percepción de Riesgos \\
\hline 2002 & Bustos, Montero y Flores & Promoción de la conservación \\
\hline 2002 & Corral & Competencias \\
\hline 2002 & Corral, Varela y González & Competencias \\
\hline 2002 & Espinosa, Orduña y Corral & Ahorro \\
\hline 2003 & Corral & Mapas cognitivos \\
\hline
\end{tabular}


Año 11.

Académica sin

Frontera

Núm. 28

ISSN: 2007-8870

\section{http://revistainvestigacionacademicasinfrontera.com}

\begin{tabular}{|c|c|c|}
\hline 2003 & Corral & Situacionales \\
\hline 2003 & Corral et al., & Creencias \\
\hline 2003 & Corral, Bechtel y Fraijo & Creencias \\
\hline 2003 & Corral, Frias y González & Percepción de Riesgos \\
\hline 2004 & Bustos & CPA \\
\hline 2004 & Bustos et al., & Habilidades de instrumentación \\
\hline 2004 & Bustos, Flores y Andrade & Sociocognición \\
\hline 2004 & Bustos, Flores, Barrientos y Martínez & Atribución y motivos \\
\hline 2004 & Corral et al., & Propensión al futuro \\
\hline 2004 & Corral et al., & Propensión al futuro \\
\hline 2004 & Corral y Pinheiro & Austeridad, altruismoy ahorro \\
\hline 2004 & Corral y Pinheiro & Conducta Sustentable \\
\hline 2004 & Corral, Fraijo y Tapía & Propensión al futuro \\
\hline 2004 & Fraijo et al., & Competencias \\
\hline 2004 & Fraijo, Tapia y Corral & Educación ambiental \\
\hline 2004 & Hernández y Reimel & Redes de cooperación y solidaridad \\
\hline 2004 & Medina et al., & Clima de relaciones \\
\hline 2004 & Valenzuela et al., & Austeridad \\
\hline 2005 & Commins y Chambers & Situación residencial \\
\hline 2005 & Fraj y Martínez & Sentimientos de comunidad \\
\hline 2005 & García & Situacionales \\
\hline 2005 & García & Emociones \\
\hline 2005 & García & Uso sustentable \\
\hline 2006 & Bechtel et al., & Creencias \\
\hline 2006 & Corral y Frias & Normatividad \\
\hline 2006 & García & Programa Educativo \\
\hline 2006 & McFarie y Hunt & Activismo \\
\hline 2006 & Milfont y Gouvea & Valores \\
\hline 2007 & García & Aproximaciones \\
\hline 2008 & Bolzan & Confianza y compromiso \\
\hline 2010 & Bizer & Credibilidad \\
\hline 2010 & Brenner & Gobernanza \\
\hline 2010 & García & Creencia \\
\hline 2010 & Gissi y Soto & Tequio y Guatza \\
\hline 2011 & Carreón et al., & Conflictos locales \\
\hline 2011 & García & Antropocentrismo \\
\hline 2011 & García & Preocupación \\
\hline 2011 & García & Valoración \\
\hline 2011 & García & Sustentabilidad \\
\hline 2012 & García & Encuadre \\
\hline 2012 & García et al., & Actitudes \\
\hline 2012 & García et al., & Estilos \\
\hline
\end{tabular}


ISSN: 2007-8870

\section{$\underline{\text { http://revistainvestigacionacademicasinfrontera.com }}$}

\begin{tabular}{|c|c|c|}
\hline 2013 & García & Mercadocracia \\
\hline 2013 & García et al., & Percepciones de riesgo \\
\hline 2013 & García y Bustos & Tarifas \\
\hline 2014 & Carreón et al., & Políticas hídricas \\
\hline 2014 & Carreón et al., & Encuadre tarifario \\
\hline 2014 & Carreón et al., & Intención de voto \\
\hline 2014 & Carreón, García y Morales & Coadministración \\
\hline 2014 & Carreón, Hernández y García & Encuadre de condonaciones \\
\hline 2014 & García et al., & Desabastecimiento \\
\hline 2015 & Carreón et al., & Ambientalismo \\
\hline 2015 & García et al., & Fiabilidad \\
\hline 2015 & García et al., & Especificación \\
\hline 2015 & García et al., & Agenda \\
\hline 2015 & García, Carreón y Quintero & Gobernanza \\
\hline 2016 & García et al., & Establecimiento de agenda \\
\hline 2016 & García et al., & Representaciones sociales \\
\hline 2016 & Pérez et al., & Dispositivos \\
\hline 2017 & García et al., & Expectativas \\
\hline 2017 & Rivera Carreón y García & Cogestión \\
\hline
\end{tabular}

Fuente: Elaboración propia

En su primera etapa que va de 1980 a 1990, los estudios de las disposiciones hídricas se centraron en los fenómenos grupales y colectivos como el activismo derivado de un procesamiento automático de información (García et al., 2012), para después en la década de 1990 a 2000 centrar su interés en el análisis del contexto y escenarios desde procesos cognitivos generales como la probabilidad de la elaboración, la necesidad de cognición, la disonancia cognitiva, el comportamiento planificado o la adopción de tecnología que; en la siguiente década de 2000 a 2010 se correlacionarían con las variables sociocognitivas más específicas como las percepciones de riesgo, los motivos de ahorro, las competencias ambientales, las habilidades y las intenciones de ahorro (García, 2007).

En su fase actual de 2010 a 2018, los estudios de las disposiciones hídricas se vinculan con fenómenos sociopolíticos como el establecimiento de la agenda (Rivera, Carreón y García, 2017), la resiliencia, la orientación hacia la sustentabilidad, la co-participación, 


\section{http://revistainvestigacionacademicasinfrontera.com}

la cogestión y la corresponsabilidad, englobándose en la gobernanza de la sustentabilidad hídrica local (García, 2010).

No obstante, los estudios de las disposiciones hídricas sólo han asociado los factores en modelos y establecido sus trayectorias de relaciones de dependencia sin considerar la importancia de la formación civil, académica y profesional con orientación hacia la sustentabilidad (García, 2011).

\section{Método}

Se llevó a cabo un estudio documental con una selección de fuentes indexadas a repositorios de Iberoamérica como Dialnet, Latindex, Redalyc y Scielo, considerando el periodo de publicación de 1980 a 2018 y la inclusión de palabras clave.

Una vez establecidos los ejes y temas de discusión se realizó una investigación cualitativa con una selección de informantes de la sociedad civil trabajadora acerca de sus disposiciones hídricas, utilizando la técnica Delphi.

Tabla 1. Descriptivos de la muestra

\begin{tabular}{llllll}
\hline Informante & Sexo & Edad & Escolaridad & Ingreso & Antigüedad \\
\hline Lavacoches & Femenino & 19 & Secundaria & 310 USD & 2,3 años \\
\hline Garrafonero & Masculino & 16 & Primaría & 345 USD & 1,8 años \\
\hline Pipero & Masculino & 24 & Secundaria & 351 USD & 1,9 años
\end{tabular}

Fuente: Elaborada con los datos del estudio 


\section{$\underline{\text { http://revistainvestigacionacademicasinfrontera.com }}$}

Se construyó una matriz de análisis de contenidos, discursos y narrativas con la finalidad de extraer el significado de las disposiciones hídricas para la formación ambiental.

La información se procesó siguiendo la técnica Delphi: síntesis de datos, contextualización de información, comparación de escenarios, integración de contenidos e inferencia de significados.

\section{Resultados}

La Figura 1 muestra la inferencia de los significados de discursos y narrativas con respecto a las disposiciones hídricas seleccionadas por los tres informantes claves.

El significado de las disposiciones hídricas para la formación ambiental parece emerger de una política de oferta y una representación de la abundancia sumamente controlada por las capacidades y habilidades de los informantes. Se trata de un proceso automático de símbolos y significados en torno a secuencias de abastecimiento y consumo relativos a la venta directa o indirecta del agua como un bien comercial.

Pues bien, en la parte sintética de los discursos es posible advertir el aprendizaje de las habilidades que permiten la distribución del recurso entre quienes demandan el servicio de abastecimiento o suministro. El énfasis en el aprendizaje de las demandas y a partir de ellas el uso del agua supone una relación simétrica entre quienes venden el agua y quienes la consumen. 


\section{http://revistainvestigacionacademicasinfrontera.com}

Figura 1. Significado de las disposiciones hídricas para la formación ambiental

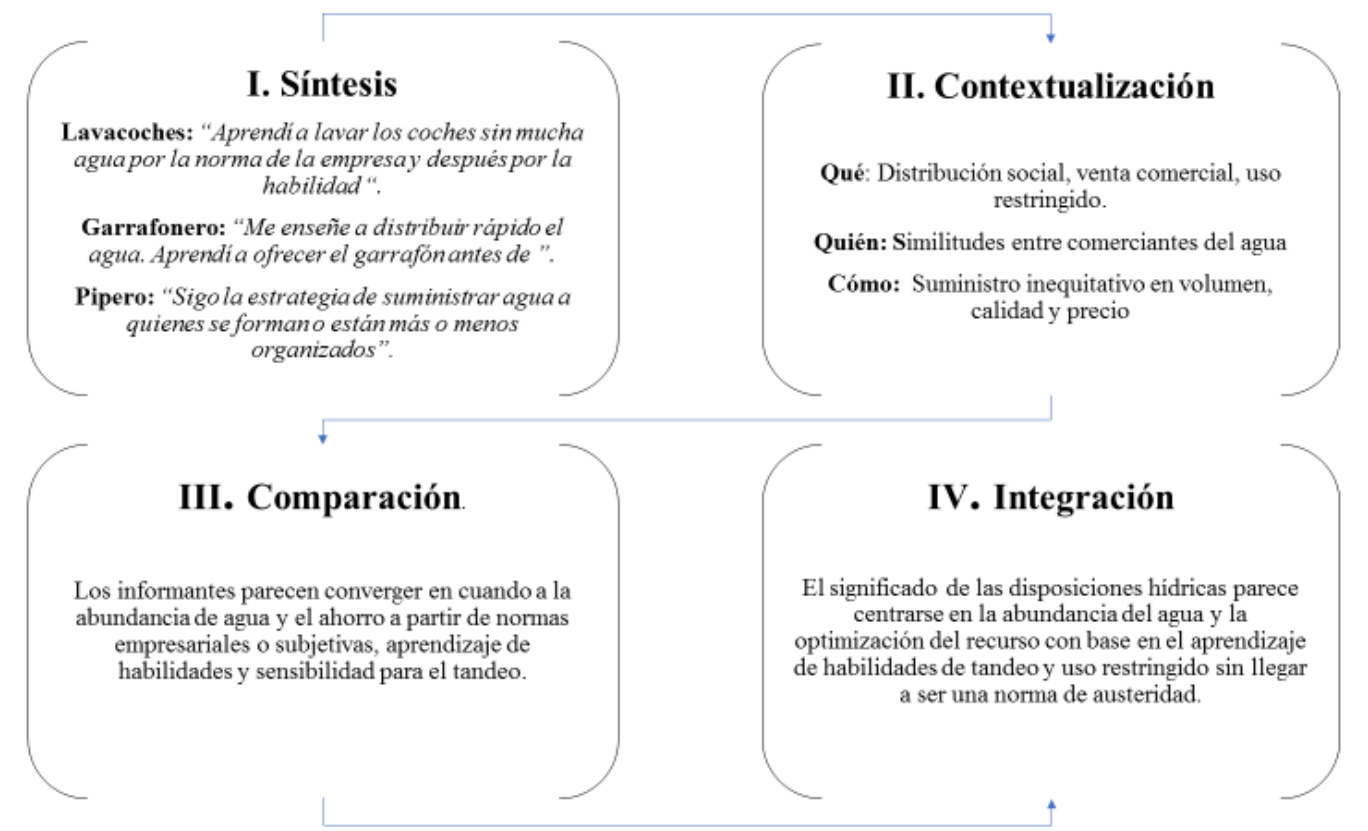

Fuente: Elaborada con los datos del estudio

En los contextos hídricos, las narrativas seleccionadas parecen develar una cadena de imágenes que ilustran una automatización de la venta, distribución y consumo del agua como si fuese un bien con valor de uso y cambio sin importar el grado de confort y recreación que supone su uso abundante. Incluso en aquellos casos de austeridad, el agua como bien social de uso y valor intangible parece orientarse hacia el sentido de intercambio de un bien que cada vez es más escaso, aún y cuando quienes la distribuyen o venden asuman que lo contrario.

En la comparación de los símbolos y significados de los informantes o entrevistados parece haber un nulo sentido de comunidad en torno a la recreación y el confort hídrico 


\section{http://revistainvestigacionacademicasinfrontera.com}

ampliamente observado en la centralidad urbana, pero que en la periferia citadina más bien se edifica un sentido de escasez, desabastecimiento y crisis.

En la parte de la integración de los discursos seleccionados es posible advertir que los entrevistados coinciden en cuanto a la optimización de recursos frente a la abundancia más que a la escasez percibida del agua, así como por el uso abundante o restringido que depara a 1 consumidor.

\section{Discusión}

El aporte del presente trabajo al estado de la cuestión radica en la develación de un significado particular de tres informantes con respecto a sus disposiciones hídricas y en relación a la formación ambiental que supone la convergencia de discursos hacia la optimización de recursos.

García (2018) interpretó los discursos en torno a la calidad de abastecimiento para comprender las narrativas en torno a la gratuidad del agua como servicio público, aunque los imaginarios son diferentes entre los ciudadanos y autoridades con respecto a la calidad del servicio que, si bien para ambos es baja, los usuarios más que las autoridades aseguran que prevalece la corrupción como problema esencial.

En el presente trabajo se ha identificado a la optimización como el núcleo central de las representaciones en torno a la venta del agua. La presunción de un bien comercial parece resignificar las habilidades de ahorro, pero en el plano opuesto de asumir que el agua es un bien común sería posible advertir el confort y la recreación hídrica.

Esto es así porque las disposiciones hídricas parecen ser un proceso que regula la información disponible contraponiéndola a su tendencia. Es decir, una difusión intensiva de abundancia de agua propiciaría un ahorro si las demandas del entorno son 


\section{http://revistainvestigacionacademicasinfrontera.com}

exacerbadas, pero si la difusión es más bien de una escasez, entonces se genera una disposición a la desesperanza que antecede al derroche ya que, los usuarios asumen que, si el agua es escasa, entonces sólo quedará disfrutar su consumo.

Pues bien, futuras líneas de investigación concernientes a la interpretación de discursos de grupos ambientalistas convencidos de la escasez del agua complementará los hallazgos del presente trabajo.

\section{Conclusión}

El objetivo del presente trabajo fue interpretar los discursos relativos a las disposiciones hídricas para comprender las narrativas alusivas a la formación ambiental, pero la literatura advierte que la simbolización de la optimización del agua sería opuesta si el agua se asumiera como un bien común. De acuerdo con la perspectiva enunciada, una aproximación de los recursos hídricos como bienes comunes supone el aprendizaje de habilidades de derroche si las demandas son mínimas.

\section{Referencias}

Bechtel, R., Asai, M., Corral, V. y González, A. (2006). A cross cultural study of environmental beliefs structures in USA, Japan, México and Peru. International Journal of Psychology. 41, 145-151

Bechtel, R., Corral, V. \& Pinheiro, J. (1999). Environmental belief systems United Status, Brazil and México. Journal of Cross-Cultural Psychology. 30, 122-128

Bustos, J. (2004). Modelo de conducta proambiental para el estudio de la conservación de agua potable. Tesis de Doctorado, Facultad de Psicología, Universidad Nacional Autónoma de México.

Bustos, J. Flores, M. y Andrade, P. (2004). Predicción de la conservación de agua a partir de factores socio cognitivos. Medio Ambiente y Comportamiento Humano. $5,53-70$ 


\section{http://revistainvestigacionacademicasinfrontera.com}

Bustos, J. y Flores, L. (2000). Evaluación de problemas ambientales, calidad del ambiente y creencias de afectación de la salud. La Psicología Social en México. 8, 445-451.

Bustos, J., Flores, L. y Andrade, P. (2002). Motivos y percepción de riesgos como factores antecedentes a la conservación de agua en la Ciudad de México. La Psicología Social en México. 9, 611-617.

Bustos, J., Flores, L., Barrientos, C. y Martínez, J. (2004). Ayudando a contrarrestar el deterioro ecológico: atribución y motivos para conservar agua. La Psicología Social en México. 10, 521-526.

Bustos, J., Montero, M. y Flores, L. (2002). Tres diseños de intervención antecedente para promover conducta protectora del ambiente. Medio Ambiente $y$ Comportamiento Humano. 3, 63-88.

Carreón, J., Bustos, J. M., García, C., Hernández, J. y Mendoza, D. (2015). Utilización de SPSS y AMOS en un estudio del pensamiento ambientalista y las intenciones de voto en una muestra de estudiantes. Multidisciplina, 20, 76-95.

Carreón, J., García, C., Morales, M. L. (2014). Hacia una administración consensuada de los recursos hídricos en ecociudades. Interdisciplinaria, 31 (1), 163-174.

Carreón, J., García, C., Morales, M. L. y Domínguez, G. A. (2011). Teorías psicosociales para explicar los conflictos derivados del abastecimiento de agua en México, Distrito Federal. Pampedia, 8, 56-68.

Carreón, J., Hernández, J. y García, C. (2014). Análisis de notas de prensa respecto a condonaciones por pago de servicios hídricos. Doxa, 47 (8), 134-152

Carreón, J., Hernández, J., García, C., Bustos, J. M., Morales, M. 1. y Aguilar, J. A. (2014). La psicología de la sustentabilidad hídrica: Políticas públicas y modelos de consumo. Aposta, 63, 1-29.

Carreón, J., Hernández, J., García, C., Rivera, B. L. y Morales, M. L. (2014). Análisis de notas de prensa en torno al encuadre sociopolítico de tarifas hídricas. Obets, 9 (1), 73-94.

Carreón, J., Hernández, J., Morales, M. L., Rivera, B. 1. y García, C. (2014). Intenciones de voto a favor de propuestas verdes ¿Nuevo paradigma ambiental y posmaterialismo social? Revista de Psicología Política, 12 (32), 37-54.

Comins, C, y Chambers, S. (2005). Psychological and situational influenses on conmuter transport mode choice. Environment and Behavior. 37, 640-66. 


\section{$\underline{\text { http://revistainvestigacionacademicasinfrontera.com }}$}

Corral, V y Frías, M. (2006). Personal normative beliefs, antisocial behavior, and residential water conservation. Environment and Behavior. 38, 406-421

Corral, V. (1997). Un análisis crítico del concepto "actitudes" parte 1: postulados y métodos de estudio. Revista Mexicana de Análisis de la Conducta. 23, 215-235.

Corral, V. (1998). Interacciones ambiente / conducta: algunas áreas de investigación. En V. M. Alcaraz y A. Bouzas (coord.). Las aportaciones mexicanas a la psicología. (pp. 55-70) México: UNAM.

Corral, V. (2000). La definición del Comportamiento Proambiental. La Psicología Social en México. 8, 466-472.

Corral, V. (2001). Aplicaciones del Modelamiento Estructural a la investigación psicológica. Revista Mexicana de Psicología. 18 193-209.

Corral, V. (2002a). A structural model of proenvironmental competency. Environment \& Behavior. 34, 531-549.

Corral, V. (2002b). Avances y limitaciones en la medición del comportamiento proambiental. en J. Guevara, y S. Mercado, (coords.) Temas Selectos de Psicología Ambiental. (pp. 483-510). México: UNAM-GRECO-UNILIBRE.

Corral, V. (2002c). Structural Equation Modelling. in Bechtel, R y Churcman, A. (eds.) Handbook of Environmental Psychology. (pp. 256-270). New York: Wiley \& Sons, Inc.

Corral, V. (2003a). ¿Mapas cognitivos o conductas ambientales? en E. Díaz, y M. Anaya, (coord.). Perspectivas sobre el cognitivismo en psicología. (pp. 37-79). México: UNAM (Iztacala).

Corral, V. (2003b). Determinantes psicológicos o situacionais do comportamento de conservaçào de àgua: um modelo estructural. Estudos de Psicología. 8, 245-252.

Corral, V. (2003c). Situational and personal determinants of waste control practices in Northern Mexico: a study of reuse and recycling behaviors. Recourses, Conservation \& Recycling. 39,265-281.

Corral, V. y Encinas, L. (2001). Variables disposicionales, situacionales y demográficas en el reciclaje de metal y papel. Medio Ambiente y Comportamiento Humano. 2, $1-19$. 


\section{http://revistainvestigacionacademicasinfrontera.com}

Corral, V. y Obregón, F. J. (1992). Modelos predictores del comportamiento proambientalista. Revista Sonorense de Psicología. 6, 5-14.

Corral, V. y Pinheiro, J. (1999). Condicoes para o estudo do comportamento próambiental. Estudos de Psicología. 4, 7-22.

Corral, V. y Pinheiro, J. (2004). Aproximaciones al estudio de la conducta sustentable. Medio Ambiente y Comportamiento Humano. 5, 1-26.

Corral, V. y Zaragoza, F. (2000) Bases sociodemográficas y psicológicas de la conducta de reutilización: Un Modelo estructural. Medio Ambiente y Comportamiento Humano. 1, 9-29.

Corral, V., Bechtel, R. y Fraijo, B. (2003). Environmental beliefs and water conservation: an empirical study. Journal of Environmental Psychology. 23, 247257.

Corral, V., Bechtel, R., Armendáriz, L. I. y Esquer, A. B. (1997). La estructura de las creencias ambientales en universitarios mexicanos: el Nuevo Paradigma Ambiental. Revista Mexicana de Psicología. 14, 173-181.

Corral, V., Capdevielle, F., Garibaldi, L. y Encinas, 1. (1986). Estrategias conceptuales para la reducción de consumo doméstico de agua en una zona urbana. La Psicología Social en México. 1, 475-479.

Corral, V., Fraijó, B. y Tapía, C. (2004). Propensiones psicológicas en niños de sexto grado de primaria. Validez de un instrumento. Anuario de Investigaciones Educativas. 7, 31-44.

Corral, V., Fraijo, B., Frías, M., González, D. y Pinheiro, J. (2004). Propensión al presente, al pasado y al futuro y sus relaciones con el ahorro de agua. La Psicología Social en México. 10, 547-552

Corral, V., Frías, M. y González, D. (2001). On the relationship between antisocial and anti-environmental behaviors: an empirical study. Population and Environment. 24, 273-286.

Corral, V., Frías, M. y González, D. (2003). Percepción de riesgos, conducta proambiental y variables demográficas en una comunidad de Sonora, México. Región y Sociedad. 26, 49-72. 


\section{http://revistainvestigacionacademicasinfrontera.com}

Corral, V., Garibaldi, L. y Encinas, L. (1987). Estudio exploratorio de patrones conductuales de consumo doméstico de agua en zona urbana. Revista Sonorense de Psicología. 2, 87-93.

Corral, V., Varela, C. y González, D. (2002). Una taxonomía funcional de competencias proambientales. La Psicología Social en México. 9, 592-597.

Espinosa, G., Orduña, V. y Corral, V. (2002). Modelamiento estructural de las competencias proambientales para el ahorro de agua. La Psicología Social en México. 9, 605-610.

Fraijo, B. S., Tapia., C. y Corral, V. (2004). Efectos de un programa de Educación Ambiental en el desarrollo de Competencias Pro ecológicas. La Psicología Social en México. 10, 539-546.

García, C. (2004). La psicología ambiental del agua en el año 2025. Bricolage, 6, 19-27

García, C. (2005). ¿Cuáles son los determinantes psicológicos y situacionales de un uso sustentable del agua en la Zona Metropolitana del Valle de México? Revista de Investigación y Educación, 15, 1-44

García, C. (2005). ¿Qué sentimientos activan el recuerdo de abastecimiento y el cuidado de agua más como un elemento comunitario que como un recurso natural? Psicología, 9 (1), 1-22.

García, C. (2005). Dos modelos para explicar el uso sustentable del agua en la zona metropolitana del valle de México. Revista de Psicología Iztacala, 8 (1), 1-39.

García, C. (2005). Los estudios psicológicos de la sustentabilidad. Nneme, 6 (13), 1-63.

García, C. (2005). Un modelo para explicar el uso de agua en la Zona Metropolitana del Valle de México. Revista Internacional de Psicología, 6 (2), 1-138.

García, C. (2005). Un modelo psicosocial para promover el ahorro de agua en la zona metropolitana del valle de México. Ciencias Sociales, 11 (2), 103-109.

García, C. (2006). Un programa de uso sustentable del agua. Primera parte. Residuos, 90, 1-14.

García, C. (2006). Un programa de uso sustentable del agua. Segunda parte. Residuos, 92, 1-14. 


\section{http://revistainvestigacionacademicasinfrontera.com}

García, C. (2007). Aproximaciones al uso sustentable del agua. Aqua Forum, 11 (44), 16-20.

García, C. (2007). Un programa de conservación de agua. Entelequía, 5, 169-196

García, C. (2009). Las investigaciones causales de las ciencias sociales en torno a las problemáticas hídricas. Gaceta de Antropología, 25 (2), 1-9.

García, C. (2009). Las investigaciones correlacionales de las ciencias sociales en torno a las problemáticas hidrológicas. Barataria, 10, 141-147.

García, C. (2009). Las teorizaciones de las ciencias sociales en torno a las problemáticas hidrológicas. Entelequia, 9, 63-98.

García, C. (2010) la creencia hidrológica en Iztapalapa. Revista de Psicología Política, 8 (24), 18-34.

García, C. (2010). La exclusión hidrológica. Entelequia, 11, 41-59.

García, C. (2011). La estructura del antropocentrismo hídrico. Multidisciplina, 10, 3342.

García, C. (2011). La preocupación hidrológica en Iztapalapa. Revista de Psicología Geppu, 2 (1), 52-63.

García, C. (2011). La valoración hidrológica en Iztapalapa. Epsys, 3, 1-9.

García, C. (2011). Las estructuraciones de la sustentabilidad hídrica. Tlatemoani, 7, 127.

García, C. (2011). Las investigaciones comparativas de las ciencias sociales en torno a las problemáticas hídricas. Revista de Desarrollo local Sostenible, 4 (11), 1-12.

García, C. (2011). Las investigaciones estructurales en torno a las problemáticas hídricas. Revista de Psicología Gepu, 2 (2), 99-112.

García, C. (2012). La cobertura de la prensa en torno a denuncias, abastecimientos y emplazamientos ante una escasez de agua en Iztapalapa, México. Sociedad Hoy, 22, 995-113.

García, C. (2012). Los constructos de la sustentabilidad hídrica. Epsys, 3, 1-19 


\section{$\underline{\text { http://revistainvestigacionacademicasinfrontera.com }}$}

García, C. (2013). El encuadre periodístico de en torno a los conflictos hídricos derivados de la mercadocracia en México. Psicología para América Latina, 24, 121-155.

García, C. (2018). Interpretación de discursos en torno al subsidio de abastecimiento hídrico para la comprensión de narrativas tarifarias. Ciencias Sociales, 4 (2), 2540 .

García, C. y Bustos, J. M. (2013). Los estudios psicológicos de la sustentabilidad hídrica. Aplicaciones al sistema tarifario de consumo. Revista de Ciencias Sociales, 139, 65-90.

García, C., Aguilar, J. A., Rosas, F. J., Carreón, J. y Hernández, J. (2015). Diferencias de fiabilidad sociopolítica ante conflictos hídricos entre actores civiles. Invurnus, $10(2), 3-13$.

García, C., Bustos, J. M., Juárez, M. Rivera, B. L. y Limón, G. A. (2017). Expectativas de usuarios del servicio de agua potable en torno al abastecimiento, la calidad y las tarifas en el marco de futuras elecciones en una localidad de la Ciudad de México. Compendium, 4 (7), 45-54.

García, C., Carreón, J. y Quintero, M. L. (2015). Dimensiones de gobernanza de la sustentabilidad hídrica. Pueblos y Fronteras, 10 (20), 195-203.

García, C., Carreón, J., Bustos, J. M. y Juárez, M. (2016). Escenarios relativos al establecimiento de agenda para la gobernanza transgeneracional de los recursos y servicios hídricos. Civilizar, 16 (31), 83-112

García, C., Carreón, J., Bustos, J. M., Hernández, J. y Salinas, R. (2015). Especificación de un modelo de riesgos ambientales ante el cambio climático. Entre Ciencias, 3 (6), 73-90.

García, C., Carreón, J., Bustos, J. M., Hernández, J., Aguilar, J. A. y Rosas, F. J. (2014). Una aproximación sociopolítica al desabastecimiento, tarifas, subsidios y tandeos relativos a los servicios hídricos. Ensayos Pedagógicos, 11 (1), 73-98.

García, C., Carreón, J., Hernández, J. y Méndez, A. (2012). Sistemas de actitudes hacia la sustentabilidad hídrica. Tecsistecalt, 4, 1-12.

García, C., Carreón, J., Hernández, J., Bustos, J. M., Bautista, M., Aguilar, J. A. y Valdés, O. (2016). Social representations about tamden periurban anthropocentrism in a neighbohoord: wáter impact to of leaks in local development. Aacademia Journal of Environmental Science, 4 (6), 101-104. 


\section{$\underline{\text { http://revistainvestigacionacademicasinfrontera.com }}$}

García, C., Carreón, J., Hernández, J., Bustos, J. M., Domínguez, G. A. y Morales, M. L. (2013). La cobertura periodística en torno a los conflictos hídricos por el desabasto de agua en una demarcación de México, Distrito Federal. Multidisciplina, 14, 21-48.

García, C., Carreón, J., Hernández, J., Mejía, S., García, E. y Rosas, F. J. (2015). Hacia una agenda hídrica para la gobernanza local sustentable. Revista Internacional de Investigación en Ciencias Social, 11 (1), 130-154.

García, C., Carreón, J., Hernández, J., Montero, M. E. y Bustos, J. M. (2013). Actitudes, consumo de agua y sistemas de tarifas del servicio de abastecimiento de agua potable. Polis, 12 (34), 363-401.

García, C., Carreón, J., Mecalco, J., Hernández, J., Bautista, M. y Méndez, A. (2013). Estructura de las percepciones de riesgo en torno a la escasez y el desabasto de agua local y global. Xhimai, 8 (15), 95-118.

García, C., Carreón, J., Mendoza, D., Aguilar, J. A., Hernández, J., Mejía, S. y Estrada, F. M. (2014). Especificación de un modelo de agenda sociopolítica en torno a los conflictos hídricos y la pacificación retributiva. Obets, 9 (2). 249-265.

García, C., montero, M. E., Bustos, J. M., Carreón, J. y Hernández, J. (2013). teoría de la actitud hacia el consumo sustentable del agua. Sustentabilidades, 8, 1-12.

García, C., Montero, M., Bustos, J. M. y Carreón, J. (2012). Los estilos de vida en torno a las problemáticas hídricas. Sustentabilidades, 4, 1-11.

McFarie, B. y Hunt, L. (2006). Environmental activism in the forest sector. Social psychological, social cultural, and contextual effects. Environment and Behavior. $38,266-285$.

Milfont, T. y Duckitt, J. (2004). The structure of environmental attitudes: a firts and second cader confirmatory factor analisis. Journal of Environmental Psychology. 24, 289-303.

Milfont, T. y Duckitt, J. (2006). Preservation and utilization the structure of environmental attitutes. Medio Ambiente y Comportamiento Humano. 7, 29-50. 
ISSN: 2007-8870

\section{http://revistainvestigacionacademicasinfrontera.com}

Milfont, T. y Gouveia, V. (2006). Time perspective and values: an exploratory study of their relations to environmental attitudes. Journal of Environmental Psychology. $26,72-82$.

Obregón, F. y Zaragoza, F. (2000). La relación de tradición y modernidad con las creencias ambientales. Revista Sonorense de Psicología. 14, 63-71.

Pato, C. y Tamayo, A. (2006). Valores, creencias ambientales y comportamiento ecológico de activismo. Medio Ambiente y Comportamiento Humano. 7, 51-66.

Pato, C., Tróccoli, B. y Tamayo, A. (2002). Values and ecological behavior: an empirical study of Brazilians students. $17^{\text {th }}$ Conference of IAPS 2002.

Pérez, G., Bustos, J. M., Juárez, M., Carreón, J., Delgado, M. A. y García, C. (2016). Gobernanza del desarrollo sustentable: Modelos y dispositivos para el trabajo social de los recursos hídricos. Margen, 83, 1-13.

Rivera, B. L., García, C. y Carreón, J. (2017). Especificación de un modelo de cogestión de los servicios hídricos. Sin Frontera, 10 (25), 1-17.

Shaphores, J., Nixon, H., Ogunseitan, O \& Shapiro, A. (2006). Household willegness to reuse electronic waste. An application California. Environment and Behavior. 38, 183-208.

Valenzuela, B., Corral, V., Quijada, A., Griego, T., Ocaña, D. y Contreras, C. (2004). Predictores disposicionales del ahorro de agua: Austeridad, Altruismo y propensión al Futuro. La Psicología Social en México. 10, 527-532. 